\title{
Time-interval acquisition on a 6502-based microcomputer
}

\author{
PHILIP A. FEMANO and DONALD W. PFAFF \\ Rockefeller University, New York, New York
}

\begin{abstract}
A versatile machine language subroutine and accompanying interface circuitry enable a 6502 based computer to collect time intervals between data events that can be represented by voltage transients. Intervals within the range of 150 microsec to $13.98 \mathrm{~min}$ can be collected with a resolution of \pm 25 microsec. Electrical interface circuitry includes signal conditioning and optical isolation and also allows user-specification of threshold levels on the data line. The machine language software timing routine and sequential interval handler include a 24-bit interval timer, a 16-bit interval counter, user-specified number and length of acquisition periods, and an out-ofmemory tester. Although presented for implementation on the Apple II series of microcomputers, the transportability of the routine to other 6502-based machines is addressed.
\end{abstract}

In the course of the investigation of certain physiological phenomena, the occurrence of specific events is sometimes not as relevant as the interval of time that transpires between events. Investigation of whether a drug induces arrhythmic activity within the myocardium or the analysis of discharge pattems of neurons or muscle fibers are examples of the usefulness of measuring intervals between events. Although the measurement of an interval is conceptually trivial, its analysis can be quite laborious if more than only a few dozen intervals are accumulated manually (e.g., when intervals are measured directly from film strip records).

Personal computers have become cost-effective laboratory tools for the acquisition and analysis of data as well as for the preparation of manuscripts. They are easier to program than larger minicomputer configurations and yet maintain moderately high-speed operation, and in some cases they lend themselves better to singleuser applications that require that the microprocessor be fully dedicated to perform specific tasks, such as the acquisition of rapid time-dependent events. Some microcomputers offer graphics that are acceptable for publication.

Our specific requirement was a computer system that could process taped records of time intervals between electromyographic spike potentials, that is, interspike intervals, recorded from back muscles. The decision to implement an Apple II Plus system was based on the versatility of its input/output (I/O) structure, speed of the 6502 microprocessor, widespread availability of competitively priced software, affordable graphics, hardware reliability, and ease of use. Although the system described in this report was designed for our

The authors' mailing address is: Department of Neurobiology, Rockefeller University, 1230 York Avenue, New York, New York 10021. specific application, it is versatile enough to serve in other applications in which time intervals between events are of interest.

The measurement of time intervals by a computer can be accomplished by using either a hardware timer circuit or a software timing loop. Although hardware clocks are very useful for logging in the time of day of each analysis, timer circuits, which give the programmer access to timing resolutions of less than $1 \mathrm{msec}$, are few in number and relatively expensive, unless homebrew circuits are devised. Furthermore, the timer circuitry can draw much power from the power supply, the limit of which can be exceeded in the Apple if too many peripheral interface cards with high power requirements are installed. On the other hand, because the execution time of each machine language instruction is known (see De Jong, 1982, and Scanlon, 1980), precise timing of events can be achieved and/or controlled by software. Thus, we have elected to implement a short machine language routine that not only measures interspike intervals with excellent time resolution, but also serves as an interval counter and controls memory allocations as these intervals are accumulated. (Note that the execution times discussed here are based on a 1-MHz 6502.)

The 6502 machine language routine described in this report enables the user to input one data line and one control line (see below) for the acquisition of interspike intervals, the number of which is limited only by the amount of free random access memory (RAM). It can be adapted for use in other 6502-based computers, since the only modifications that must be made pertain to the device-dependent memory map of each model of computer. In this report, all features that are dependent upon the Apple II Plus memory map are noted in order to aid those who wish to adapt this program to other 6502-based machines. Additional versatility is achieved by the fact that the routine is relocatable throughout 
RAM as long as the proper precautions are taken to ensure that branch conditions are not executed across memory page boundaries (see Memory Map section).

Although this report is presented in a tutorial manner, it is not meant to teach assembly language to the novice and does not expect the reader to have prior facility in assembly language. It does assume that the prospective user has programming resources for a high-level language so that the appropriate tailored application software can be generated to fit individual needs. A sample application package has been developed in BASIC (see Program Availability).

\section{THE SYSTEM}

\section{Overview}

Our basic system for the acquisition of interspike intervals consists of an Apple II Plus computer (48K) with interface circuitry to the signal lines (see below), two Apple Disk II drives (one for the application software and one for mass storage of data, although a single drive is sufficient if the user is willing to let software and data co-reside on the same disk), and a 12-in. greenphosphor NEC video monitor. A printer is required for hardcopies of the data and/or graphs. Although interspike-interval analyses can be performed during realtime experimentation, we record the raw physiological data on tape for subsequent postexperimental analysis. Since the output signal from the tape recorder is bipolar analog, it first has to be converted to one that is TTL compatible. This is accomplished by passing the signal through either: (1) a commercially available window discriminator (or Schmitt trigger) whose output pulse is TTL compatible, or (2) a simple comparator/oneshot circuit designed in the laboratory (see below). The resulting signal is then suitable for acquisition and processing by the computer.

During the period of data acquisition by the computer (i.e., following signal conversion to TTL levels), two signals are presented: the control signal and the data signal. The control signal is simply a single logichigh square pulse, the duration of which specifies the length of the acquisition period during which the data signal is analyzed. The number and duration of acquisition periods can be user specified. When the control line goes to a logic-high state, the computer looks at the data line containing the data spikes. As long as the control signal is held at a logic-high level, the computer continues to accumulate interspike intervals measured from the data line. An annunciator delivers an output pulse with the detection of each data pulse in order to provide feedback from the analysis. When the control signal goes to a logic-low level, the activity in the data line is ignored. At this point, the computer may either return control to the main program (which may be written in a high-level language, such as BASIC) or start the acquisition process again by looking for another logic-high level on the control line, if one is specified during the set-up procedure. In either case, interspike intervals are stored sequentially in memory, and an interval counter increments as each interval is accumulated.

If the user elects to record the real-time data on tape for subsequent analysis, then the control signal and the data signal should be stored on separate channels.

\section{Interfacing to the $1 / 0$ Connector}

The circuitry required to interface the signal lines to the computer is shown in Figure 1. The circuit accepts analog signals with positive data spikes (or event mark ings) up to $+5 \mathrm{~V}$ and outputs TTL compatible pulses to the input port of the computer.

The purpose of the comparator is to: (1) isolate impedances so that many different devices can be used to convey the signals (e.g., a tape recorder) without attenuating the signal through the usual voltage division that occurs when impedances are not properly matched, (2) allow user-specified threshold detection of the signals, (3) eliminate any negative voltage phases in the signal lines, and (4) provide hysteresis in the ON versus OFF threshold levels in order to prevent switching noise in slow or noisy transients, such as those found in biological signals. Figure 1 uses the LM 339 quadcomparator, but the single-channel LM 311 can also serve as the first stage in each of the two signal lines.

The next stage in the interface for the data line is a 74121 TTL one-shot, which controls the width of the data pulse from the comparator to a prespecified value of 100 microsec (see below). The PULSE WIDTH potentiometer is set so that the output pulse of the 74121 is approximately 100 microsec. This value is obtained with the potentiometer adjusted to a resistance of approximately $15 \mathrm{kohms}$.

The circuit for the control line omits the 74121 oneshot stage and, instead, includes a NAND gate, which inverts the output of the comparator.

The last stage of the interface circuitry for both the data and control lines employs an optoisolator (HewlettPackard 6N135), which completely isolates the external hardware from the computer circuitry. In so doing, the user is assured that high-voltage shorts in the external circuitry do not invade the low-power circuits within the computer. Furthermore, the use of an optoisolator prevents the computer circuitry from becoming involved in ground loops.

All components within this interface circuitry receive power from the $+5 . \mathrm{V}$ supply line of the built-in 16-pin $\mathrm{I} / \mathrm{O}$ connector located at J14 on the Apple II motherboard and draws less than $80 \mathrm{~mA}$ from the power supply. In our system, the PBO line of the $I / O$ connector is the control line, and PB1 is the data line. Within the data-line circuitry, the PULSE WIDTH control on the one-shot should be adjusted so that an input transient from logic high to logic low (from the output of the comparator) results in an output pulse of 100 -microsec duration from the 74121 , regardless 


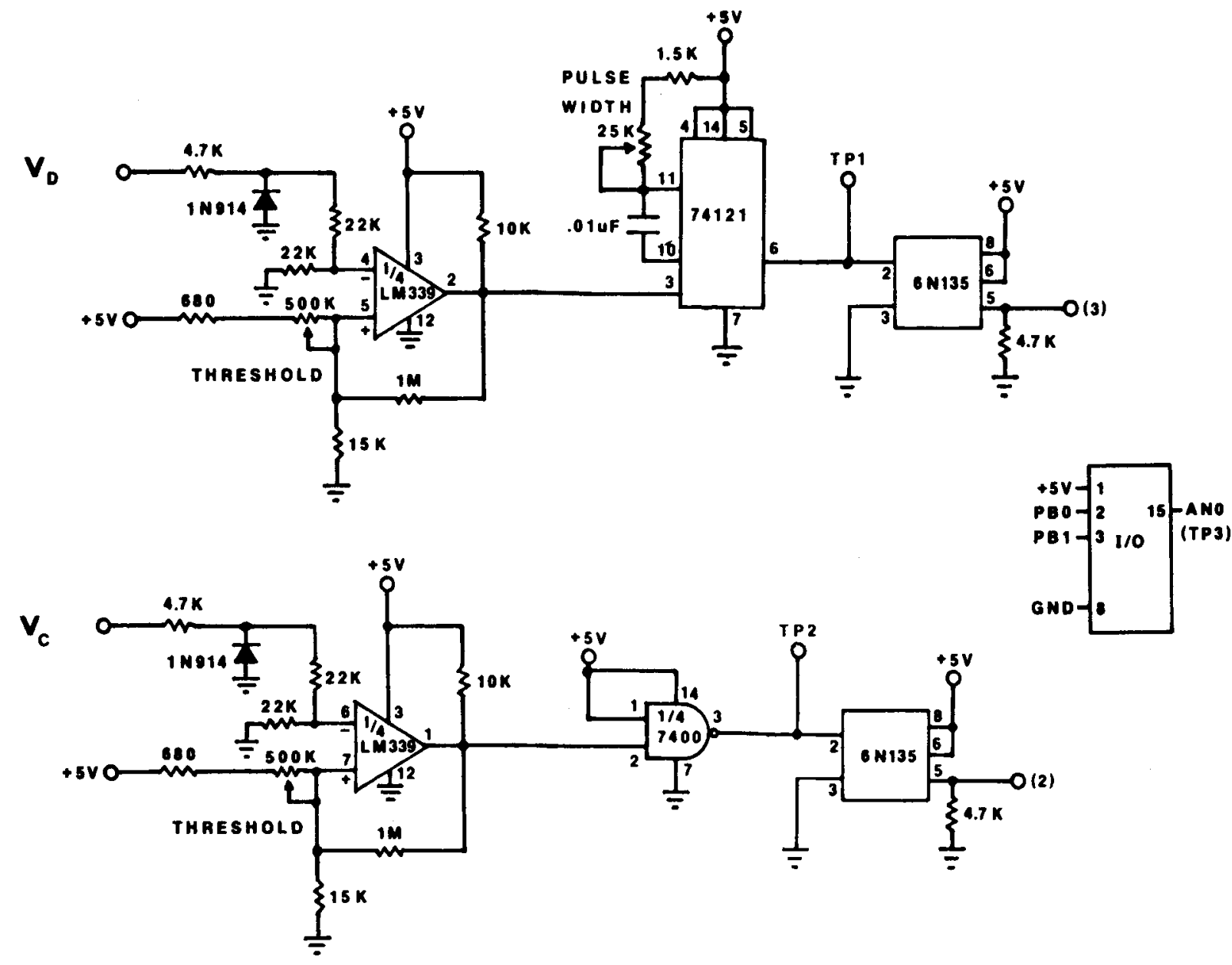

Figure 1. Interface circuitry to the I/O connector. Vd and $\mathrm{Vc}$ are the inputs from the data and control lines, respectively. Numbers in parentheses at the output of each channel refer to the pin numbers on the $/ / O$ connector (shown on the right) to which the lines should be directed. Touchpoints for the data (TP1), control (TP2), and annunciator (TP3) lines are indicated and serve to enable monitoring of each signal. See text for circuit description.

of the duration of the input pulse. Thus, the output signals from both lines of the interface are active high and TTL compatible.

\section{Software}

The short machine language subroutine is versatile enough to serve in many different applications that require the analysis of time intervals between events. Although only 146 bytes long, the routine provides the following features:

1. A 24-bit timer measures time intervals ranging from 150 microsec to $13.98 \mathrm{~min}$, with a resolution of \pm 25 microsec.

2. The length and number of data-acquisition periods are user controlled (via the control line).

3. A 16-bit interval counter is employed.

4. The number of intervals acquired can be user specified and is limited only by the amount of free RAM.
5. An annunciator output provides feedback from the analysis.

6 . The acquisition period is automatically terminated when all free RAM is filled.

The logic of the routine is shown in the flowchart of Figure 2. Note that the use of the word event instead of muscle spike is testimony to the general usefulness of the routine in obtaining high-speed measurements of time intervals between brief voltage pulses. After clearing the registers and initializing the counter, the routine verifies that a control signal is expected and then initializes the timer. Also, before the timer can start, the control signal must go to a logic-high level. The data line is then examined for a data pulse, which, in our application, represents a muscle-spike potential. The occurrence of a pulse starts the timer. The timer is incremented, and as long as the control line is high and there is no data pulse on the data line, the timer increments again, each timing loop requiring 50 microsec of 


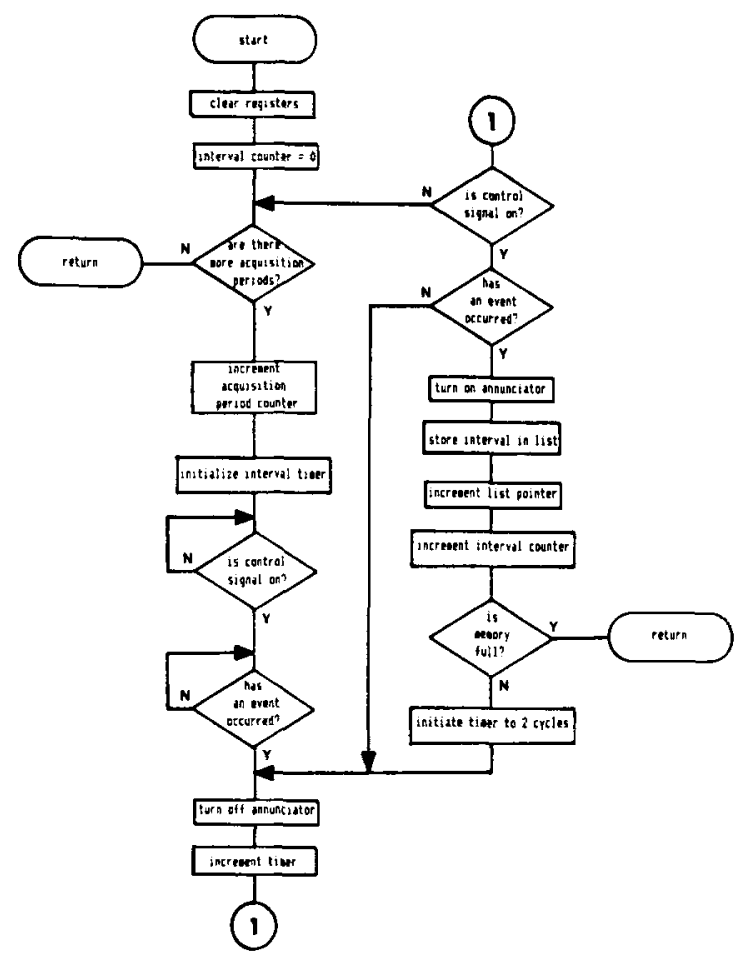

Figure 2. Flowchart of the logic of the interval-handler routine.

instruction-execution time. Thus, intervals are accumulated in 50-microsec increments. This cycling of the timing loop occurs until another data pulse is detected in the data line. When the data line pulses high, the annunciator output port also goes to a logic-high level. The 3-byte value of the 24-bit timer is stored in an interval list at a memory location determined by a list pointer, which is then updated to point to the next open memory location. An interval counter is also incremented. If the limit of free memory is reached, then control is returned to the main program. However, if free memory is not yet full, the timer is reset, and the routine tests again for the state of the control line. If the control line is still high, then the acquisition period is still active and the data line is analyzed for more activity. On the other hand, if the control line is low, but more acquisition periods are specified by the user in the set-up procedure (see below), then the timer is reinitialized and the cycle repeats itself until all control signals specified by the user have been completed or the upper limit of free memory has been reached.

The entire annotated source code is shown in Figure 3. It follows the flowchart description precisely. Statements 45 through 63 contain the 50-microsec timing loop. Annunciator output, interval storage, list pointer and interval-counter increments, memorylimit detection, and timer resetting all occur within lines 65 through 103 and contain 100 microsec of code (excluding the 1-microsec overhead for the no-branch
035F: 8

$0361=18$

0361: 18

0362 : AS FA
0364: 69 03

0364: 69 O3

0368: $25 \mathrm{FB}$

036A: 69 00
036C: O5 FB

036P: 18

036P: 18 E

0371: 6901

0373: 85 E

0375: 15 E

$0377: 6900$
$0379: 85$ EC

037B: $\mathrm{NS} \mathrm{FB}$

037B: $\mathrm{NSF}$

037D: CS FC

0381: 200

0381: 29 O0

0385: 85 r

0397: 1902

0389: 85 FD

0380: No $58 \mathrm{co}$ $0390: 58$
$0391: 60$



Figure 3. 6502 source code listing for the interval handler. 
condition in line 63). Thus, the routine measures intervals with 50 -microsec increments of the software timer (i.e., \pm 25 -microsec resolution), but only intervals exceeding 150 microsec can be measured, since this is the "duty cycle" of the timer following a data pulse. Ideally, the data pulse from the interface circuitry should have a duration greater than 50 microsec but less than 150 microsec. Pulses shorter than 50 microsec may not be detected, whereas pulses greater than 150 microsec may register multiple intervals every 150 microsec for the duration of the data pulse. These problems can be avoided by properly adjusting the PULSE WIDTH control (see Figure 1) for an output pulse of 100 microsec.

\section{Set-Up Procedure}

The software timing loop described above is meant to be called as a subroutine within an application program, which can be written in either machine language or a high-level language (see Program Availability below). As such, certain parameters must be specified by the user before the subroutine is called. These values can be entered via the keyboard in response to questions asked by a set-up module within the main program. A description of each parameter, along with an explanation of its use in the subroutine, follows:

1. Name of the data file. Assigning a name to the file created by the population of intervals about to be acquired is necessary for subsequent storage of data to a disk. This name is not handled by the subroutine itself, but is used by the main program to effect mass storage and subsequent retrieval.

2. Number of acquisition periods. The number (and duration) of control signal pulses can be specified so that the user can gate the computer into analyzing only the pertinent sequences of the data stream. Up to 256 periods can be specified. This number is tested in line 26 (see Figure 3) to determine whether additional acquisition periods are forthcoming.

3. Number of intervals. The user has the option to direct the timer subroutine to terminate and return to the calling program when the prespecified number of intervals has been acquired. The default value is equal to the maximum amount of free RAM. The number of intervals can be specified, since the upper border of free RAM is set at address 38400 , the beginning of Apple DOS. (Note-hexidecimal notation will be indicated in standard 6502 format. Thus, decimal 38400 is $\$ 9600$ in hex notation.) This limit is set by the main program by loading location $\$ F C$ with the number $\$ 96$ (decimal = 150 ) and is tested in line 95 of the listing (Figure 3). Since the interval timer is 24 -bits wide, 3 bytes are required to store the value of each interval. Thus, in order to specify the number of intervals to be measured, the main program simply multiplies this number by 3 and subtracts the product from 38400 . This resulting address is used to initialize the 16-bit list pointer in zero page locations $\$ F A$ and $\$ F B$. These locations are used to move intervals from the timer to the proper location in RAM in lines 66 through 76 of the listing. The list pointer is then incremeted by 3 in lines 78 through 84 to point to the next free memory location. If the maximum number of intervals is desired, then the list pointer should be loaded initially with the location just above HIMEM, which indicates the upper boundary of memory reserved for BASIC program variables [see Applesoft II Basic Programming Reference Manual (Apple Computer, Inc., 1978, p. 43)]. Note that care must always be taken to ensure that the designated lower limit of free RAM resides an integer multiple of 3 bytes counted down from the upper limit of free RAM (location \$9600).

\section{Memory Map and Other Device-Dependent Considerations}

The system described in this report, including software and interface circuitry, is completely compatible with all standard configurations of the Apple II series computers: Apple II, Apple IIt, and Apple IIe. Other 6502-based computers will require some degree of modification if this acquisition system is to be implemented successfully. The following discussion describes dependence of our system on the Apple II memory map and hardware configuration (Apple Computer, Inc., 1979).

The relative addressing mode used by the unconditional branch of lines 27 and 103 (see Figure 3) is used instead of a JMP statement in order to render the subroutine relocatable. Page $\$ 03$ is an ideal location for short machine language routines in the Apple II Plus, but users of other 6502-based machines may wish to load the routine elsewhere in RAM. In those cases, precautions must be made to ensure that a branch condition does not cross memory page boundaries, since this involves extra machine cycles that distort the precision of the timer. (References to this very important subtlety of the 6502 are made by De Jong, 1982, p. 60, and Scanlon, 1980, p. 57).

The two input lines as well as the annunciator output line are accessed by the built-in address decoding circuitry and are therefore hardwired in the Apple to hexadecimal locations \$C061 (PB0), \$C062 (PB1), and \$C058/\$C059 (ANO OFF/ON) and are accessed on the I/O connector at pins 2, 3, and 15, respectively (see Figure 1). Other 6502-based machines may have built-in single-bit $\mathrm{I} / \mathrm{O}$ ports similar in function to those found in the Apple. Alternatively, the interface circuitry may have to be connected to the bus through the appropriate addressdecoding circuitry. The appropriate changes in addressing should be made in the routine when used in other machines. The activation of the annunciator is provided only as feedback for the user and is of no consequence to the operation of the routine; therefore, if an annunciator output line is not available, then substituting two NOP statements where references are made to the annunciator line (i.e., lines 48 and 65) will cause an 
equivalent amount of time to transpire (i.e., 4 microsec for each line), thus maintaining the precision of the timing loop while saving 2 bytes of code.

The implementation of the timer routine requires the use of nine zero page memory locations that are not used by DOS, BASIC interpretor, or the system monitor [see Apple II Reference Manual (Apple Computer, Inc., 1979)]. The utilization of zero page ( $\$ 0000$ to $\$ 00 F F$ ) addressing modes is important to the timing of the routine, but such free address space may be difficult to find in other 6502-based machines. (Refer to the appropriate reference manuals for zero page availability.) In our system, these values are loaded into the proper memory locations using the POKE instruction of BASIC from the main program. The zero page usage is as follows:

1. The interval counter uses locations \$EB and \$EC and is automatically initialized to zero in lines 24 and 25 of the listing (Figure 3).

2. The number of acquisition periods specified by the user in the set-up procedure is loaded into location \$EF before data acquisition begins. This number is compared with a counter (line 26) that is incremented (line 28) each time the control signal goes to a logic-high level.

3. The 16-bit interval list pointer is located at \$FA and $\$ F B$ and is loaded initially with a value representing the beginning of the block of memory in which consecutively acquired intervals will be stored and that is calculated from the number of intervals specified by the user in the set-up procedure described above.

4. The upper limit of memory reserved for interval storage is at location $\$ 9600$ (decimal $=38400$ ) and represents the beginning of the disk operating system in the 48K Apple II Plus with DOS 3.3. The page number (\$96) is stored in location \$FC in the routine, and when the high byte of the list pointer reaches this value (tested in line 95), then memory is full, the routine is terminated, and all previously acquired intervals are left intact. Thus, the range of RAM used to accumulate intervals resides between HIMEM (from BASIC) and DOS. Note that, if a full 64K of RAM is implemented (e.g., with the addition of a $16 \mathrm{~K}$ peripheral RAM board in the Apple II or IIt), then DOS may be moved to this additional region of RAM, using commercially available utility routines (e.g., Bongers, 1983), and this increases the available RAM for data storage. In this case, the value held in the page pointer at zero page location $\$ F C$ should be changed appropriately to include this extra available memory.

5. The 24-bit interval timer is located at $\$ F D, \$ F E$, and \$FF. It is initialized to a value of 1 at the beginning of each acquisition period (line 29) to maintain the fidelity of the timer (the KILTIM routine at line 42 completes a 75-microsec delay so that the first timer loop covers the 50-microsec range from 75 to 125 microsec or $100 \pm 25$ microsec). Furthermore, because of the 100 -microsec required for statement-execution time during the memory management within lines 65 through
103 , it is initialized to a value of two following the acquisition of each interval (the equivalent of two main timing loops of 50 microsec each).

6. If the main application program is written in BASIC (see Program Availability), then it must be possible to load values directly into specific memory locations, examine the value of specific memory locations, and call the machine language subroutine. In APPLESOFT BASIC, this can be accomplished using the PEEK, POKE, and CALL commands. Other 6502-based machines should have a similar capability to interface a high-level language with machine language.

\section{Performance}

Figure 4 illustrates how the control signal acts as a gate in the analysis of the data. The data and control signals were taken from points TP1 and TP2, respectively, in Figure 1, and the bottom trace was taken from the ANO annunciator output at pin 15 of the $\mathrm{I} / \mathrm{O}$ connector (TP3 in Figure 1). Note that, during the acquisition period, the control signal is held high and the number of intervals acquired (numbers in bottom trace) is $I=D-P$, where $D$ is the number of data spikes that were passed to the timer routine and $P$ is the number of acquisition periods within the analysis $(\mathrm{P}=2$ in Figure 4). The interval value that accumulates from the last spike within the last acquisition period to the time when the control signal drops low is not transferred to the main interval list but is held in the 24-bit timer (at $\$ F D$, $\$ F E$, and $\$ F F$ ) when the routine is returned to the calling program, in the event that this value is needed.

A simple example of the performance of the routine can be seen in Figure 5, which shows a histogram of intervals resulting from an attempt to manually switch an externally applied $+5-\mathrm{V}$ pulse to the data line at a rate of about 3 pulses/sec. Note that the distribution centering around $333 \mathrm{msec}$ represents the imprecision of human approximation. In contrast, an analysis using a square pulse generator at approximately 3 pulses/sec (interpulse interval $=334.1 \pm 0.025 \mathrm{msec}$ ) is shown in Figure 6. In this latter example, the list pointer at \$FA and $\$ F B$ was initialized to $\$ 93 \mathrm{~A} 8($ decimal $=37800)$ in the set-up procedure, thus predetermining the acquisition of 200 intervals.

It is not necessary for the data to be recorded on tape before they can be analyzed. No change in the system described in this report is necessary in order to obtain an analysis during real-time data acquisition. The interface circuitry simply would receive its input lines directly from the experimental instrumentation amplifiers. The data shown graphically in Figure 5 were analyzed in real time. The main advantage of real-time analysis is that more immediate feedback about the data can be obtained. The main disadvantage is that a finite segment of data can be processed in a given analysis, and during real-time acquisition, there may be no way, a priori, of knowing when the important segment of data will occur. For example, it is difficult to analyze the inter- 


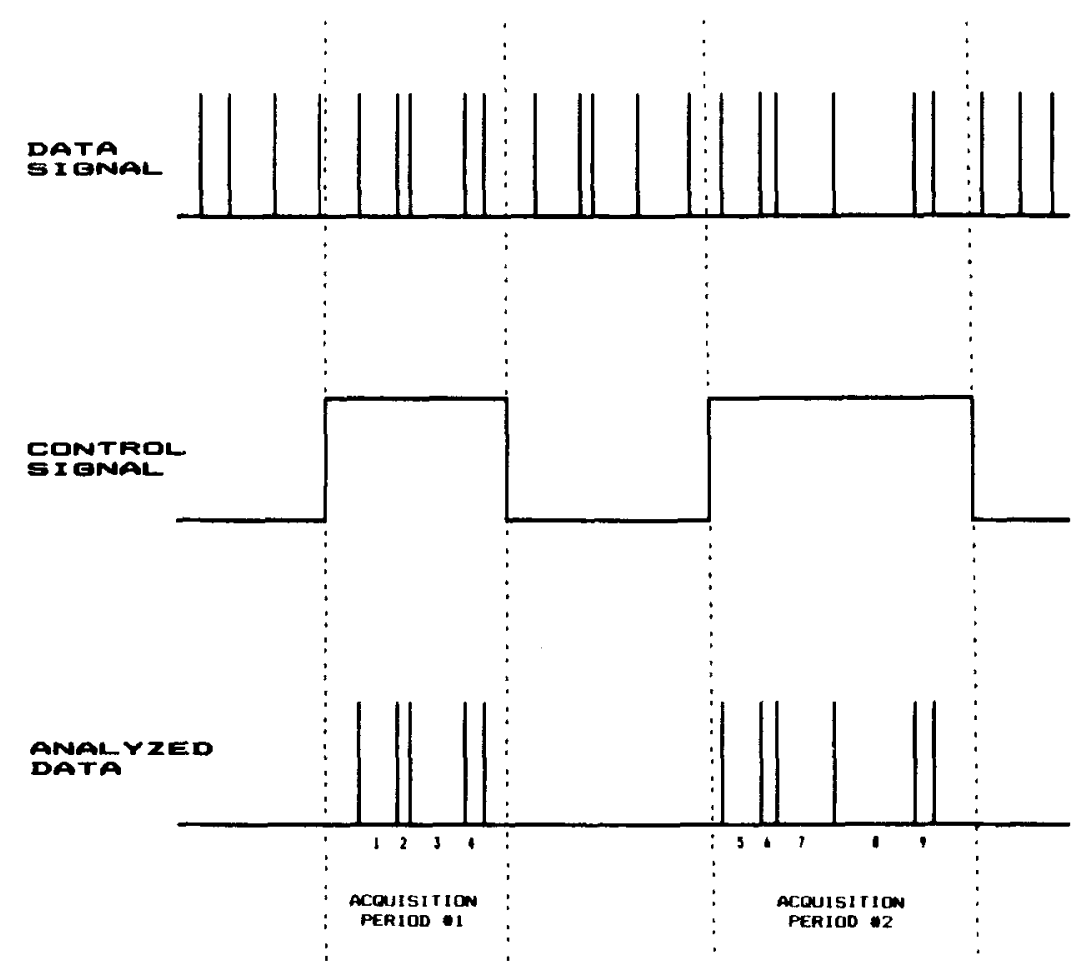

Figure 4. Logic schematic of a data-acquisition sequence using the timer routine.

spike intervals within a burst of spikes from a neuron that only infrequently exhibits this bursting pattern. Another example is the identification of the first few irregular intervals in an EKG following the administration of a drug that induces cardiac arrhythmias.

\section{Limitations}

In order to take advantage of the versatility of the routine, it is necessary to establish the limitations of its performance. The total free RAM available is limited by the size of the application program and the RAM-based DOS (unless hardware memory expansion has been implemented). Our version of the main program easily allowed enough RAM for 5,000 intervals (almost 15K of RAM). Thus, with a stream of 200-microsec intervals, only $1.0 \mathrm{sec}$ of data can be analyzed. On the other hand, if the data signal consists of a continuous stream of pulses at $13.98-\mathrm{min}$ intervals, then 48.54 days of data can be analyzed! However, since most applications involve signals containing intervals of assorted lengths,

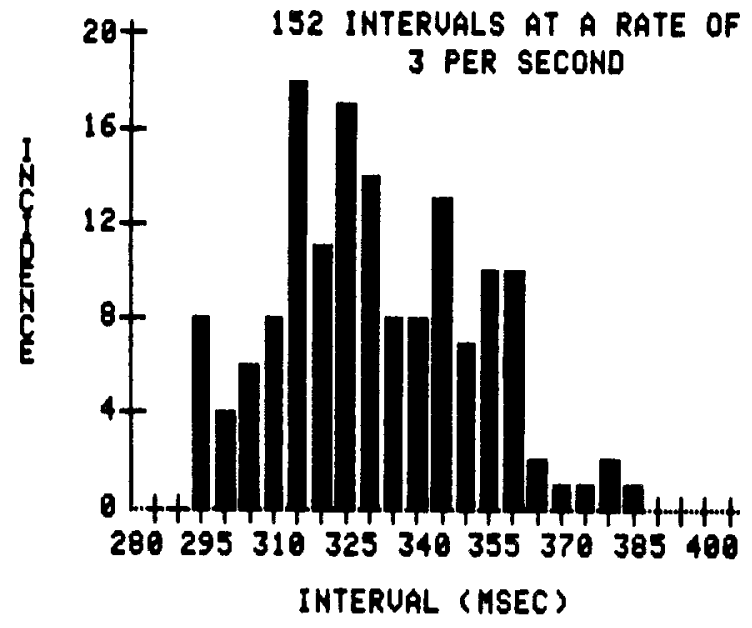

Figure 5. Response-interval histogram of the author's performance at pushing a switch at a rate of $3 / \mathrm{sec}$.

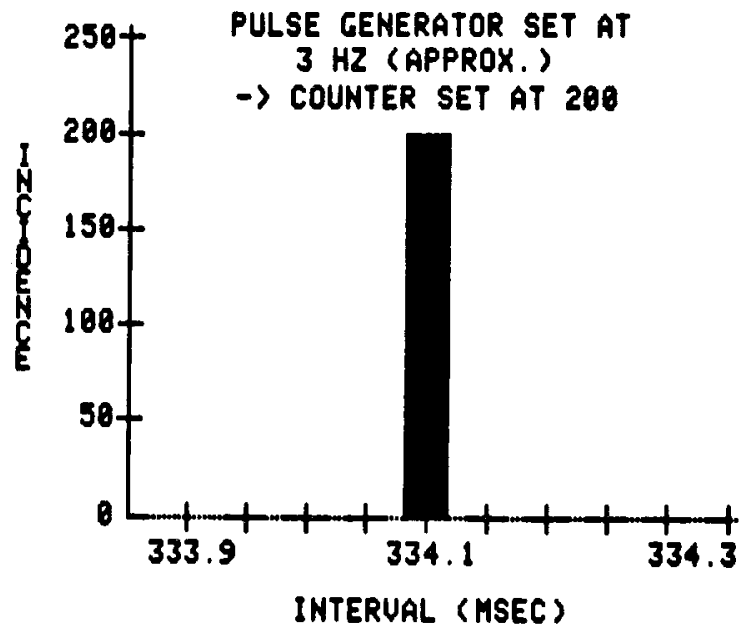

Figure 6. Histogram of a train of pulses at approximately $3 \mathrm{~Hz}$ delivered by a pulse generator. 
the maximum possible acquisition time will vary within these extremes.

As stated above, the optimal data pulse width should be between 50 and 150 microsec. However, the data pulse going into the interface circuitry can be virtually any duration, since the pulse width exiting the circuitry is set to 100 microsec by the PULSE WIDTH potentiometer (see Figure 1).

Following the first data pulse at the beginning of an acquisition period, in order for the timer to be incremented and the data line to be tested again, the control signal must remain high for at least 116 microsec. This restriction is imposed mainly because of the 75 -microsec delay loop at lines 37 through 43 . Also, since the control line is tested once within the 50 -microsec timing loop, it should remain low for at least 50 microsec in order to detect reliably the end of an acquisition period.

Since the control line is tested before the data line (lines 60 and 62 in the listing), there is a possibility that a data pulse occurring 6 microsec after the control line drops to a logic-low level will be included in the analysis. This should not present a problem, since it is well within the \pm 25 -microsec resolution specified for the routine.

Internupts requested through the IRQ line are disabled by the timer routine, since the software timing loop loses meaning if an interrupt is serviced while intervals are accumulating. However, before exiting the routine, the CLI instruction clears the interrupt disable flag and allows subsequent IRQ signals to be handled.

\section{DISCUSSION}

We have described a general-purpose high-performance machine language routine for the measurement of intervals between the onset of voltage-marked events. Thus, although our specific application was to measure interspike intervals of electromyographic recordings (Femano, Schwartz-Giblin, \& Pfaff, in press), it has also been used for the analysis of neural data (Kow \& Pfaff, 1983; Schwartz-Giblin, Halpern, \& Pfaff, Note 1) and can be used to measure intervals between the onset of virtually any two or more events that can be represented electrically within the parameters discussed. For example, the routine is suited for accumulating behavioral reflex latencies or response patterns (e.g., Figure 5 in this report).

The version presented here can measure intervals from 150 microsec to $13.98 \mathrm{~min}$. If intervals longer than this are expected, then the timing loop can be lengthened by adding more time-consuming statements within the loop and/or the timer itself can be expanded to more than 24 bits. The latter method, although technically more difficult, is preferable because each additional bit length of the timer will extend the maximum measurable interval by approximately a power of two while increasing the minimum measurable interval by only a few microseconds.
Since all of the intervals are stored sequentially in RAM, many different analyses can be performed on the same set of data. For example, the total acquisition time that transpires between the first and last data pulse within an acquisition period can be obtained simply by adding up all of the time intervals. In addition, various time-dependent characteristics of the interval population can be analyzed. Furthermore, if a switch is installed to bypass the 74121 one-shot in Figure 1 (in addition to adding a NAND gate as the middle stage similar to that in the control line interface), then the PULSE WIDTH controller is bypassed. In this way, a data pulse with a duration exceeding 150 microsec will register multiple 150-microsec intervals for the duration of the spike. This can be useful in determining the duration of brief events (e.g., milliseconds) and in comparing the lengths of different events with each other and with the intervals that transpire between them.

Although the microcomputer represents a relatively new tool for the neuroscientist, its usefulness within a laboratory environment cannot be overstated. Already, applications have been reported for digitizing field potential data (Teyler, Mayhew, Chrin, \& Kane, 1982), graphic reproduction of neurohistochemical maps (Williams \& Elde, 1982), and three-dimensional reconstruction of serial sections (Prothero \& Prothero, 1982). As these instruments become easier to use, to program, and to afford, more neuroscientists will have a means to develop and implement new experimental designs and revealing methods of data analysis.

\section{PROGRAM AVAILABILITY}

We have developed a software package written in BASIC that is available as a listing at no charge or on disk (DOS 3.3) for a nominal fee. This package does not include APPLEPLOT graphics, which is a commercially available graphics package from Apple Computer, Inc. Our software package does include a short program that converts the interval files into APPLEPLOT-compatible files.

\section{REFERENCE NOTE}

1. Schwartz-Giblin, S., Halpern, M., \& Pfaff, D. W. Segmental organization in lordosis behavior: EMG and muscle nerve recordings. Manuscript submitted for publication, 1983.

\section{REFERENCES}

Apple Computer, Inc. Applesoft II Basic programming reference manual. Cupertino, Calif: Author, 1978.

Apple Computen, Inc. Apple II reference manual. Cupertino, Calif: Author, 1979.

Bongers, C. Moving DOS to the RAM card. In V. J. Golding (Ed.), All about DOS. Kent, Wash: Apple Pugetsound Program Library Exchange, 1983.

De Jong, M. L. Apple II assembly language. Indianapolis, Ind: Sams, 1982.

Femano, P. A., Schwartz-Giblin, S., \& Pfaff, D. W. Brainstem reticular influences on lumbar axial muscle. American Journal of Physiology, in press, 1983. 
Kow, L.-M., \& Praff, D. W. Suprachiasmatic neurons in brain slices of ovariectomized rats: Electrophysiological and neuropharmacological characterizations and the effects of chronic estrogen treatment. Brain Research, in press, 1983.

Prothero, J., \& Prothero, J. Three-dimensional reconstruction from serial sections. I. A portable microcomputer-based software package in Fortran. Computers and Biomedical Research, $1982,15,598-604$.

SCAnlon, L. J. 6502 software design. Indianapolis, Ind: Sams, 1980.
Teyler, J. T., Mayhew, W., Chrin, C., \& Kane, J. Neutophysiological field potential analysis by microcomputer. Journal of Neuroscience Methods, 1982, 5, 291-303.

Williams, F. G., \& ELDE, R. A microcomputer-aided system for the graphic reproduction of neurohistochemical maps. Computer Programs in Biomedicine, 1982, 15, 93-102.

(Manuscript received August 16, 1983;

revision accepted for publication September 29, 1983.) 